\title{
Correlation Filters for Pattern Recognition Using a Noisy Reference
}

\author{
Pablo M. Aguilar-González and Vitaly Kober \\ Department of Computer Science, Centro de Investigación Científica y de Educación \\ Superior de Ensenada, \\ Km. 107 Carretera Tijuana-Ensenada, Ensenada 22860, B.C., México \\ \{paguilar, vkober\}@cicese.mx \\ http://www.cicese.mx/
}

\begin{abstract}
Correlation filters for recognition of a target in overlapping background noise are proposed. The object to be recognized is given implicitly; that is, it is placed in a noisy reference image at unknown coordinates. For the filters design two performance criteria are used: signalto-noise ratio and peak-to-output energy. Computer simulations results obtained with the proposed filters are discussed and compared with those of classical correlation filters in terms of discrimination capability.
\end{abstract}

Keywords: correlation filters, pattern recognition.

\section{Introduction}

Since the pioneering work by VanderLugt [1], correlation filters have been extensively studied for the purpose of pattern recognition [2-15]. Within the context of pattern recognition, detection and location estimation are two very important tasks. When a correlation filter is used, such tasks may be solved in two steps; that is, first, the detection is carried out by searching the highest correlation peaks at the filter output, and then the coordinates of the peaks are taken as position estimations of targets in the scene image [2].

Different criteria have been proposed to evaluate the performance of correlation filters [3] such as signal-to-noise ratio (SNR), peak sharpness, light efficiency, discrimination capability, etc. Filters are designed by maximizing one of these criteria. Many filters have been proposed when an input scene contains a target distorted by additive noise. The matched filter (MF) [1] is derived by maximizing the SNR. The phase-only filter 44 maximizes light efficiency. The optimal filter (OF) 5] minimizes the probability of anomalous errors. Several filters have been derived for the non-overlapping scene model 66781911. The generalized matched filter [7] was derived by maximizing the ratio of the square of the expected value of the correlation peak to the average output variance. The generalized optimum filter [7] maximizes the peak-to-output energy ratio (POE). Recently [15], several correlation filters were proposed for the scene model that takes into account linear degradations of the both scene and target.

All of these filters, however, are derived under the assumption that a target is explicitly known. However, in real-life situations the target is often given by 
a reference image, which contains the reference object at unknown coordinates, as well as a noisy background. The considered signal model is close to practical situations, in which observed and reference images are inevitably corrupted by noise owing to the image formation process. In this paper, we propose two correlation filters optimized with respect to the SNR and POE. The performance of the filters is compared to that of common correlation filters.

\section{Analysis}

We use the additive signal model for the input scene and the reference image. For simplicity, 1-dimensional notation is used and it is assumed that the target is located at the origin of the reference image. Formally, the scene and reference image are given, respectively, by

$$
\begin{aligned}
& s(x)=t\left(x-x_{0}\right)+n_{s}(x), \\
& r(x)=t(x)+n_{r}(x),
\end{aligned}
$$

where $t(x)$ is the target, $s(x)$ is the observed scene with the target location $x_{0}, r(x)$ is the reference image, and $n_{s}(x)$ and $n_{r}(x)$ are noise signals in the input scene and the reference image, respectively. Both $n_{s}(x)$ and $n_{r}(x)$ are assumed to be stationary random processes. It is also assumed that the random processes and the random target location $x_{0}$ are statistically independent of each other. $S(\omega)$ and $T(\omega)$ are the Fourier transforms of $s(x)$ and $t(x)$, respectively, and $N_{s}(\omega)$ and $N_{r}(\omega)$ are the power spectral densities of $n_{s}(x)$ and $n_{r}(x)$, respectively.

Since the target signal is not available, we look for a correlation filter of the following form:

$$
H(\omega)=A(\omega) R^{*}(\omega),
$$

where $A(\omega)$ is a deterministic function, $R(\omega)$ is the Fourier transform of the realization of the reference image given in (2), and * denotes complex conjugate. Actually, it is interesting to note that the filter is given by a bank of the transfer functions determined by a realization of the random process $n_{r}$.

The additive-robust matched filter (AR-MF) can be derived by maximizing the SNR criterion:

$$
\mathrm{SNR}=\frac{\left|\mathrm{E}\left\{y\left(x_{0}\right)\right\}\right|^{2}}{\operatorname{Var}\left\{y\left(x_{0}\right)\right\}},
$$

where $\mathrm{E}\{\cdot\}$ denotes the expected value and $\operatorname{Var}\{\cdot\}$ denotes variance. The expected filter output at the target location can be calculated as

$$
\mathrm{E}\left\{y\left(x_{0}\right)\right\}=\frac{1}{2 \pi} \int A(\omega) E\left\{R^{*}(\omega) S(\omega) e^{i \omega x_{0}}\right\} d \omega .
$$

The variance of the output is given by

$$
\operatorname{Var}\left\{y\left(x_{0}\right)\right\}=\frac{1}{2 \pi} \int|A(\omega)|^{2} \operatorname{Var}\left\{R^{*}(\omega) S(\omega)\right\} d \omega .
$$


Using (11) and (2) to calculate (5) and (6), we obtain the following expression for the SNR:

$$
\mathrm{SNR}=\frac{\left|\int A(\omega)\left(|T(\omega)|^{2}+\mu_{s} T(0) \delta(\omega)\right) d \omega\right|^{2}}{2 \pi \int|A(\omega)|^{2}\left(|T(\omega)|^{2}\left(N_{r}(\omega)+N_{s}(\omega)\right)+N_{r}(\omega) N_{s}(\omega)\right) d \omega},
$$

where $\mu_{s}$ is the mean of the scene noise and $\delta(\omega)$ is the Dirac delta function. Applying the Cauchy-Schwartz inequality we obtain the expression for the ARMF

$$
H_{A R-M F}(\omega)=\frac{|T(\omega)|^{2}+\mu_{s} T(0) \delta(\omega)}{|T(\omega)|^{2}\left(N_{r}(\omega)+N_{s}(\omega)\right)+N_{r}(\omega) N_{s}(\omega)} R^{*}(\omega) .
$$

The additive-robust optimum filter (AR-OF) is derived by maximizing the POE criterion:

$$
\mathrm{POE}=\frac{\left|\mathrm{E}\left\{y\left(x_{0}\right)\right\}\right|^{2}}{\mathrm{E}\left\{\overline{|y(x)|^{2}}\right\}}
$$

where the over-bar denotes spatial averaging, i.e. $\overline{y(x)}=(1 / L) \int y(x) d x, L$ is the spatial extent of the signal $y(x)$. We can calculate $\mathrm{E}\left\{\overline{|y(x)|^{2}}\right\}$ as

$$
\mathrm{E}\left\{\overline{|y(x)|^{2}}\right\}=\frac{1}{2 \pi L} \int|A(\omega)|^{2} \mathrm{E}\left\{\left|R^{*}(\omega) S(\omega)\right|^{2}\right\} d x
$$

Substituting (5) and (10) into (9) we get

$$
\mathrm{POE}=\frac{\left|\int A(\omega)\left(|T(\omega)|^{2}+\mu_{s} T(0) \delta(\omega)\right) d \omega\right|^{2}}{\frac{2 \pi}{L} \int|A(\omega)|^{2}\left(|T(\omega)|^{4}+|T(\omega)|^{2}\left(N_{r}(\omega)+N_{s}(\omega)\right)+N_{r}(\omega) N_{s}(\omega)\right) d x},
$$

and applying the Cauchy-Schwartz inequality, the expression for the AR-OF is given by

$$
H_{A R-O F}(\omega)=\frac{|T(\omega)|^{2}+\mu_{s} T(0) \delta(\omega)}{|T(\omega)|^{4}+|T(\omega)|^{2}\left(N_{r}(\omega)+N_{s}(\omega)\right)+N_{r}(\omega) N_{s}(\omega)} R^{*}(\omega) .
$$

Note that if the reference image does not contain noise, the AR-MF and the $\mathrm{AR}-\mathrm{OF}$ are equal to the classical MF and OF, respectively.

It can be seen that the obtained filters require knowledge of the target power spectrum. This contradicts the assumption that information about the target is unknown. However, with the help of the used model the power spectrum of the target can be estimated from the reference power spectrum as follows:

$$
|T(\omega)|^{2}=|R(\omega)|^{2}-N_{r}(\omega) .
$$

Because images are real and non-negative, we are also able to estimate $T(0)$ as $T(0)=\sqrt{|T(0)|^{2}}$. 


\section{Computer Simulations}

In this section we present computer simulation results. The performance of the proposed filters are compared with that of the classical filters in terms of the discrimination capability (DC). The DC is formally defined [5] as the ability of a filter to distinguish a target from other objects in the scene. The DC can be expressed as follows:

$$
D C=1-\frac{\left|C^{B}(0)\right|^{2}}{\left|C^{T}(0)\right|^{2}},
$$

where $\left|C^{B}(0)\right|$ is the maximum value in the correlation plane over the background area, and $\left|C^{T}(0)\right|$ is the maximum value in the correlation plane over the area the target occupies in the input scene. The background area and the target area are complementary. Values of the DC close to unity, indicate a good capacity to discriminate the target against unwanted objects. Negative values of the $\mathrm{DC}$ indicate a failure to detect the target.

The size of all images used in the experiments is $256 \times 256$ pixels. All filters are implemented using the Discrete Fourier Transform. The intensity values are in the range [0-255]. We use the butterfly shown in Fig. 1(a) as a target. There are two background types, shown in Fig. 1] deterministic and stochastic backgrounds. The stochastic background is a realization of a colored random process with the mean and standard deviation of 10 and 20, respectively, and with the horizontal and vertical correlation coefficients of 0.5 . To guarantee statistically correct results, 30 statistical trials of each experiment for either different positions of the target or realizations of random processes were performed. It is worth noting in every test case in which the target is correctly detected, the target location is accurately estimated.

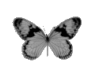

(a)

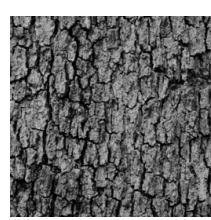

(b)

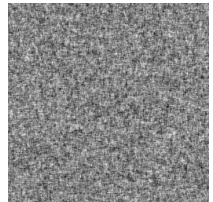

(c)

Fig. 1. (a) The target used in the experiments, (b) deterministic background, (c) example of stochastic background

\subsection{Scenario 1}

In order to compare the performance of the proposed filters, first we performed experiments when the background and a realization of the reference noise were fixed, while the location of the target within the scene was varied. The simulation results are shown in Fig. 2, It can be seen that the performance for the MF and $\mathrm{OF}$ remains almost constant. It is because these filters are not affected by the reference noise. It can also be seen that for the stochastic background, 


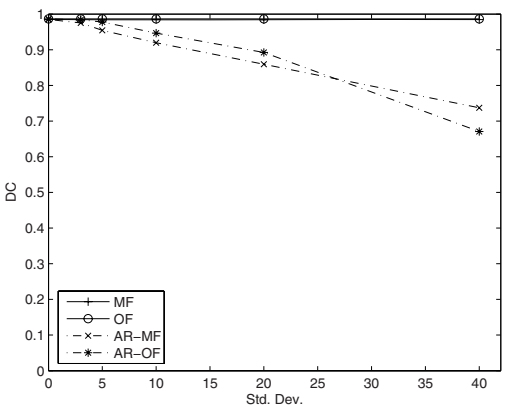

(a)

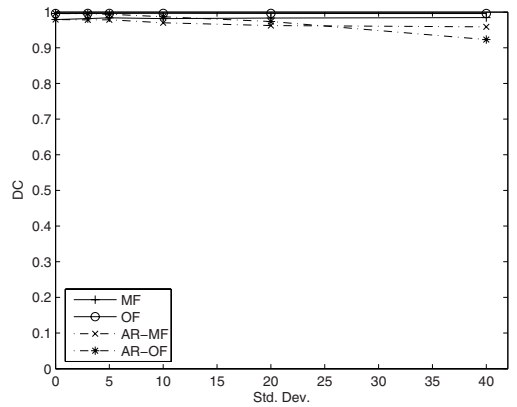

(b)

Fig. 2. Performance of correlation filters for scenario 1 with (a) deterministic background, (b) stochastic background

the performance of the proposed filters remains stable and degrades slightly only in high-noise situations. In the case of the deterministic background, the performance drop of the proposed filters is more noticeable but the target is still accurately detected.

\subsection{Scenario 2}

Since the proposed filters are given by a bank of the transfer functions determined by a realization of the reference noise process, it is of interest to evaluate the robustness of the filters to different realizations of the reference noise. In order to evaluate this, we carried out experiments in which the background and the location of the target were fixed, while the reference noise images were varied. The results are shown in Fig. 3. In this scenario the performance of the MF and OF remains constant because they are not affected by the reference noise. For the proposed filters, the mean value of the DC slightly decreases. However, there were no failures to detect the target.

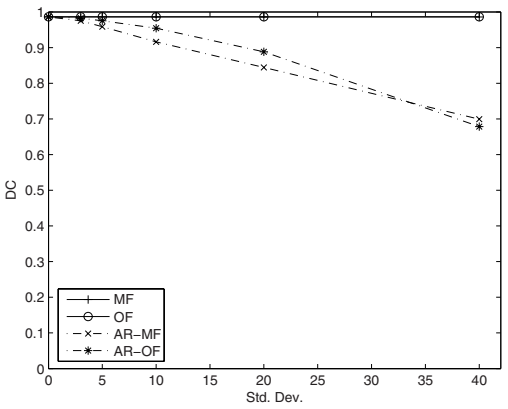

(a)

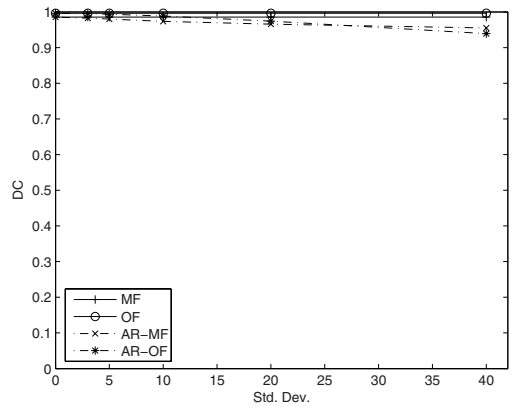

(b)

Fig. 3. Performance of correlation filters for scenario 2 with (a) deterministic background, (b) stochastic background 


\subsection{Scenario 3}

In order to determine the robustness of the proposed filters to variations in all of the random variables, we carried out experiments in which the background, target location and reference noise were varied. For this scenario, we performed 100 experiments for each value of the standard deviation. The simulation results are shown in Fig. 4. Note that the performance of the proposed filters in this scenario coincides with the results of scenarios 1 and 2 . Therefore, the performance of the proposed filters does not depend on a particular instance of the reference noise. Moreover, because the number of experiments is sufficient, with a given level of confidence, for each value of the standard deviation, we can calculate the confidence interval of the DC.

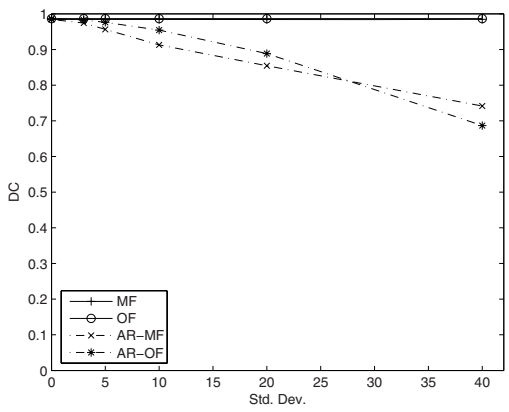

(a)

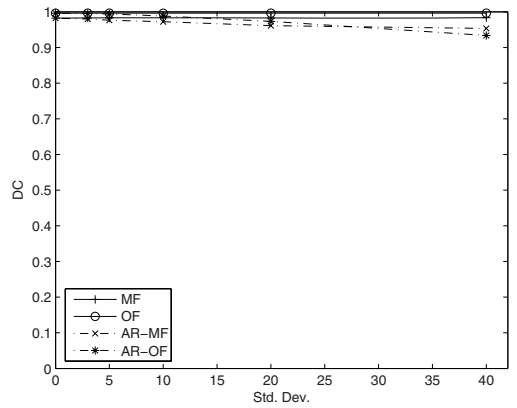

(b)

Fig. 4. Performance of correlation filters for scenario 3 with (a) deterministic background, (b) stochastic background

\subsection{Scenario 4}

Next we design approximate matched and optimum filters by substituting the noisy reference instead of the target. It is expected that the presence of noise should affect the filter performance. In this scenario we compare the performance the proposed filters with that of the approximate filters. For this set of experiments, we take a fixed background while the location of the target and the reference image noise are varied. The results are shown in Fig. 5] When a deterministic background is used, the performance of the approximated filters drops quickly as the variance of the noise increases. The approximated OF completely fails to detect the target when the standard deviation of the reference noise is high. The proposed filters, however, prove to be robust to the presence of noise and accurately locate the target in all tests. For the stochastic background, the performance of the approximated filters does not drop significantly. However, the proposed filters perform better than the approximated ones when the level of the reference noise increases. 


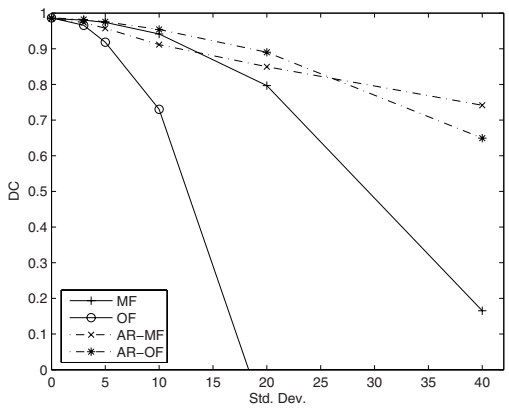

(a)

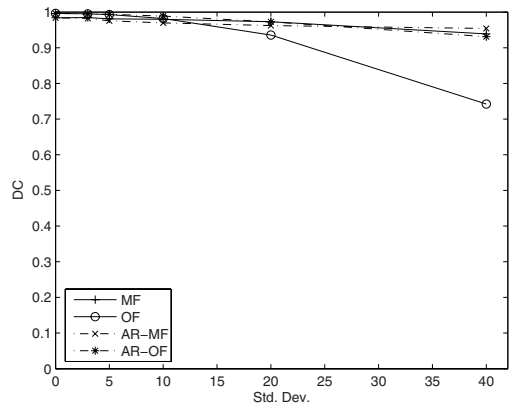

(b)

Fig. 5. Performance of correlation filters for scenario 4 with (a) deterministic background, (b) stochastic background

\section{Conclusion}

In this paper new correlation filters for recognition of an unknown target in overlapping background noise were proposed. The filters are derived from a new reference model, which takes into account the presence of additive noise in the reference image. With the help of computer simulations, we showed that the proposed filters yield good results even in the presence of high levels of noise. Moreover, the proposed filters are robust to different realizations of the reference image noise.

\section{References}

1. VanderLugt, A.B.: Signal Detection by Complex Filtering. IEEE Trans. Inf. Theory IT 10, 139-145 (1964)

2. Vijaya-Kumar, B.V.K., Mahalanobis, A., Juday, R.D.: Correlation Pattern Recognition. Cambridge U. Press, Cambridge (2005)

3. Vijaya-Kumar, B.V.K., Hassebrook, L.: Performance Measures for Correlation Filters. Appl. Opt. 29, 2997-3006 (1990)

4. Horner, J.L., Gianino, P.D.: Phase-Only Matched Filtering. Appl. Opt. 23, 812-816 (1984)

5. Yaroslavsky, L.P.: The Theory of Optimal Methods for Localization of Objects in Pictures. In: Wolf, E. (ed.) Progress in Optics, vol. 33, pp. 145-201. Elsevier, Amsterdam (1993)

6. Javidi, B., Wang, L.: Optimum Filter for Detection of a Target in Nonoverlapping Scene Noise. Appl. Opt. 33, 4454-4458 (1994)

7. Javidi, B., Wang, J.: Design of Filters to Detect a Noisy Target in Nonoverlapping Background Noise. J. Opt. Soc. Am. A 11, 2604-2612 (1994)

8. Réfrégier, P., Javidi, B., Zhang, G.: Minimum Mean Square Error Filter for Pattern Recognition With Spatially Disjoint Signal and Scene Noise. Opt. 18, 1453-1455 (1993) 
9. Javidi, B., Horner, J.L.: Real-Time Optical Information Processing. Academic Press, Inc., London (1994)

10. Vijaya-Kumar, B.V.K., Dickey, F.M., DeLaurentis, J.M.: Correlation Filters Minimizing Peak Location Errors. J. Opt. Soc. Am. A 9, 678-682 (1992)

11. Kober, V., Campos, J.: Accuracy of Location Measurement of a Noisy Target in a Nonoverlapping Background. Journal OSA 13, 1653-1666 (1996)

12. Kober, V., Ovseyevich, A.: Phase-Only Filter with Improved Filter Efficiency and Correlation Discrimination. Pattern Recognition and Image Analysis 10, 514-519 (2000)

13. Diaz-Ramirez, V.H., Kober, V., Álvarez-Borrego, J.: Pattern Recognition With an Adaptive Joint Transform Correlator. Appl. Opt. 45, 5929-5941 (2006)

14. González-Fraga, J.A., Kober, V., Álvarez-Borrego, J.: Adaptive Synthetic Discriminant Function Filters for Pattern Recognition. Opt. Eng. 45, 057005, 1-10 (2006)

15. Ramos, E.M., Kober, V.: Design of Correlation Filters for Recognition of Linearly Distorted Objects in Linearly Degraded Scenes. J. Opt. Soc. Am. A 24, 3403-3417 (2007)

16. Jenkins, J.M., Watts, D.G.: Spectral Analysis and its Applications. Holden-Day, Inc. (1968)

17. Dougherty, E.R.: Random Processes for Image and Signal Processing. Wiley-IEEE Press (1998) 